\title{
Arakelov theory of even orthogonal Grassmannians
}

\author{
Harry Tamvakis*
}

\begin{abstract}
We study the Arakelov intersection ring of the arithmetic scheme OG which parametrizes maximal isotropic subspaces in an even dimensional vector space, equipped with the standard hyperbolic quadratic form. We give a presentation of the ring $\mathrm{CH}(\overline{\mathrm{OG}})$ (when $\mathrm{OG}(\mathbb{C})$ is given its natural invariant hermitian metric) and formulate an 'arithmetic Schubert calculus' which extends the classical one for the cohomology ring of OG. Our analysis leads to a computation of the Faltings height of $O G$ with respect to its fundamental embedding in projective space, and a comparison of the resulting formula with previous ones, due to Kaiser and Köhler $[\mathrm{KK}]$ and the author [T3], [T4].
\end{abstract}

Mathematics Subject Classification (2000). Primary 14G40; Secondary 14M15, 05E0.

Keywords. Arakelov theory, orthogonal Grassmannian, characteristic classes, Schubert calculus, heights.

\section{Introduction}

In this paper we continue the author's study of the arithmetic intersection theory of projective schemes $\mathfrak{X}$ defined over the ring of integers whose fiber at infinity is a homogeneous space of a complex Lie group. The theory is most explicit when $\mathfrak{X}(\mathbb{C})$ is a hermitian symmetric space of compact type. In this case, the Arakelov Chow group $\mathrm{CH}(\overline{\mathfrak{X}})$ of Gillet and Soulé [GS1] admits a natural ring structure, which is the focus of our attention here.

The compact irreducible hermitian symmetric spaces have been classified by É. Cartan [C]. Among them, the most interesting families are the type A Grassmannians and the maximal isotropic Grassmannians in the other classical Lie types. The papers [GS2], [Ma], [T2] and [T3] studied the Arakelov Chow ring of Grassmannians (in type A) and the Lagrangian Grassmannian (in type C), respectively. We consider here the case of the even orthogonal Grassmannian $\mathrm{OG}=\mathrm{OG}(n+1,2 n+2)$, which parametrizes (one family of) isotropic subspaces of dimension $n+1$ in a $(2 n+2)$ dimensional vector space equipped with a smooth quadratic form, over any base field.

\footnotetext{
*The author was supported in part by NSF grants DMS-0296023 and DMS-0401082.
} 
Although similar to the Lagrangian case, as we shall see, the analogous theory for OG is substantially more difficult.

The key ingredient used in these works is the theory of characteristic classes for algebraic vector bundles equipped with hermitian metrics [BC], [GS2]. Indeed, there is a tautological short exact sequence of hermitian vector bundles over OG

$$
\bar{\varepsilon}: 0 \longrightarrow \bar{S} \longrightarrow \bar{E} \longrightarrow \bar{Q} \longrightarrow 0,
$$

and the arithmetic Chern classes $\hat{c}_{i}$ of $\bar{Q} \cong \bar{S}^{*}$ together with the harmonic forms on $\mathrm{OG}(\mathbb{C})$ generate $\mathrm{CH}(\overline{\mathrm{OG}})$. We now have to deal with the following three main problems: (i) find a presentation of $\mathrm{CH}(\overline{\mathrm{OG}})$ in terms of generators and relations, (ii) determine an arithmetic Giambelli formula which gives polynomials in the generators which represent 'arithmetic Schubert classes', and (iii) describe algorithms for computing the structure constants in the multiplication table of $\mathrm{CH}(\overline{\mathrm{OG}})$; this is the arithmetic Schubert calculus (compare with [KT, §1])

If $\Lambda_{n+1}$ denotes the ring of symmetric functions in $n+1$ variables, then we have an arithmetic characteristic class map

$$
\Phi: \Lambda_{n+1} \longrightarrow \mathrm{CH}(\overline{\mathrm{OG}}) ; \quad f \longmapsto \hat{f}\left(\bar{S}^{*}\right) .
$$

The crucial fact is that $\Phi$ is multiplicative; this implies that the same polynomials in the Chern classes of $S^{*}$ that solve the Giambelli problem in cohomology may be used to define the arithmetic Schubert classes. Moreover, since $\Phi$ is an algebra homomorphism, it may be used, together with a presentation of the Arakelov Chow ring $\mathrm{CH}(\overline{\mathrm{OG}})$, to understand the products of Schubert classes in $\mathrm{CH}(\overline{\mathrm{OG}})$, following [T2], [T3].

The added difficulty here is that there is an extra relation in the standard presentation of $\mathrm{CH}(\mathrm{OG})$, when compared to the Chow ring of the Lagrangian Grassmannian. According to Borel [Bo], the ring $\mathrm{CH}(\mathrm{OG})$ is generated by the Chern classes of $S$ modulo the relations (i) $c(S) c\left(S^{*}\right)=1$ and (ii) $c_{n+1}\left(S^{*}\right)=0$. In the arithmetic setting, the Whitney sum relation (i) becomes

$$
\hat{c}(\bar{S}) \cdot \hat{c}\left(\bar{S}^{*}\right) \equiv 1+(0, \tilde{c}(\overline{\mathcal{E}})),
$$

where $\tilde{c}(\overline{\mathcal{E}})$ is a Bott-Chern form for the exact sequence $\overline{\mathcal{E}}$, which may be evaluated as in the works cited previously. However, the second relation implies that the arithmetic top Chern class $\hat{c}_{n+1}\left(\bar{S}^{*}\right)$ is the class of a harmonic differential form in $\mathrm{CH}(\overline{\mathrm{OG}})$, whose computation is more challenging. To solve this problem, we first show that the desired form is a constant $r_{n}$ times the class of the Chern form $c_{n}(\bar{S})$. The exact value of $r_{n}$ is determined in $\S 5$, by comparing the formula for the Faltings height of OG derived from the arithmetic Schubert calculus with the more complicated expression for the same height in [T4, Thm. 6]. We note that the latter formula was obtained 
using a result of Kaiser and Köhler [KK], proved by completely different methods, in addition to our work [T3] in the Lagrangian case.

The results of this paper were announced at the International Conference on Arakelov Geometry in Luminy in May of 2002. The author is grateful to Vincent Maillot and Christophe Soulé for their efforts in organizing this stimulating event. Thanks are also due to Ira Gessel and Guoce Xin for their help with the proof of the hypergeometric identity in Proposition 2, and to the anonymous referee for a careful reading of the manuscript.

\section{The Arakelov Chow ring $\mathrm{CH}(\overline{\mathrm{OG}})$}

Let $k$ be any field, $E$ a vector space over $k$ of dimension $2 n+2$, and let $e_{1}, \ldots, e_{2 n+2}$ be a basis of unit coordinate vectors in $E$. Define a hyperbolic quadratic form $q$ on $E$ by setting, for any vector $v=\sum x_{i} e_{i}$,

$$
q(v)=x_{1} x_{2 n+2}+x_{2} x_{2 n+1}+\cdots+x_{n+1} x_{n+2} .
$$

The scheme of $q$-isotropic subspaces of maximal dimension $n+1$ splits into 2 connected components, which are $\mathrm{SO}(2 n+2)$ orbits; subspaces $V$ and $V^{\prime}$ lie in the same orbit (or family) if $\operatorname{dim}\left(V \cap V^{\prime}\right) \equiv(n+1)(\bmod 2)$. The orthogonal Grassmannian $\mathrm{OG}=\mathrm{OG}(n+1,2 n+2)$ is the scheme which is isomorphic to the component containing $\operatorname{Span}\left\{e_{1}, \ldots, e_{n+1}\right\}$, over any base field $k$. This is a smooth Chevalley scheme over $\operatorname{Spec} \mathbb{Z}$, which admits a cellular decomposition induced by the Bruhat decomposition of $\mathrm{SO}(2 n+2)$ (see e.g. [J, §13]).

We also let $E$ denote the trivial vector bundle of rank $2 n+2$ over OG and $S$ the rank $n+1$ tautological subbundle of $E$. Using the quadratic form $q$, we can identify the quotient bundle $E / S$ with $S^{*}$. We thus have a universal short exact sequence

$$
\varepsilon: 0 \longrightarrow S \longrightarrow E \longrightarrow S^{*} \longrightarrow 0
$$

of vector bundles over OG.

For any abelian group $M$, we let $M_{\frac{1}{2}}=M \otimes_{\mathbb{Z}} \mathbb{Z}\left[\frac{1}{2}\right]$. According to Borel [Bo], the Chow ring $\mathrm{CH}(\mathrm{OG})_{\frac{1}{2}}$ is presented as a quotient of the polynomial ring in the Chern classes of $S^{*}$ modulo the relations

$$
\text { (i) } c(S) c\left(S^{*}\right)=1, \quad \text { (ii) } c_{n+1}\left(S^{*}\right)=0 \text {. }
$$

Relation (i) comes from the Whitney sum formula applied to $\mathscr{E}$, while (ii) may be understood as follows. Let $H$ be a hyperplane in $E$ such that the restriction of $q$ to $H$ is non-degenerate. As $H$ contains no isotropic subspace of dimension $n+1$, the sequence of vector bundles over $\mathrm{OG}$

$$
0 \longrightarrow S \cap H \longrightarrow S \longrightarrow E / H \longrightarrow 0
$$


is exact. Since the line bundle $L=E / H$ is trivial, we deduce that $c_{n+1}(S)=$ $c_{n}(S \cap H) c_{1}(L)=0$ in $\mathrm{CH}(\mathrm{OG})$.

Let $\mathrm{x}=\left\{\mathrm{x}_{1}, \ldots, \mathrm{x}_{n+1}\right\}$ denote the Chern roots of $S^{*}$. Each symmetric polynomial $\phi$ in the $\left\{\mathrm{x}_{i}\right\}$ corresponds to a characteristic class $\phi\left(S^{*}\right)$ in $\mathrm{CH}(\mathrm{OG})$. In terms of the root variables $\left\{\mathrm{x}_{i}\right\}$, observe that relation (i) above may be written as $\prod_{i}\left(1-\mathrm{x}_{i}^{2}\right)=1$. It follows that we can express $\mathrm{CH}(\mathrm{OG})_{\frac{1}{2}}$ as a quotient of the ring $\mathbb{Z}_{\frac{1}{2}}\left[\mathrm{x}_{1}, \ldots, \mathrm{x}_{n+1}\right]^{S_{n+1}}$ modulo the relations

$$
\text { (i) } e_{k}\left(\mathrm{x}^{2}\right)=0,1 \leqslant k \leqslant n, \quad \text { (ii) } e_{n+1}(\mathrm{x})=\mathrm{x}_{1} \cdots \mathrm{x}_{n+1}=0 \text {, }
$$

where $e_{k}\left(\mathrm{x}^{2}\right):=e_{k}\left(\mathrm{x}_{1}^{2}, \ldots, \mathrm{x}_{n+1}^{2}\right)$ denotes an elementary symmetric function in the squares of the root variables. Note that not all of the above root variables are necessary in order to obtain a presentation. Indeed, setting $\mathrm{x}_{n+1}=0$ realizes $\mathrm{CH}(\mathrm{OG})_{\frac{1}{2}}$ as a quotient of the ring $\mathbb{Z}_{\frac{1}{2}}\left[\mathrm{x}_{1}, \ldots, \mathrm{x}_{n}\right]^{S_{n}}$ modulo the relations $e_{k}\left(\mathrm{x}_{1}^{2}, \ldots, \mathrm{x}_{\mathrm{n}}^{2}\right)=0$, for $1 \leqslant k \leqslant n$. However, the 'extra' root variable will be important in the arithmetic setting.

We next give an analogous presentation of the Arakelov Chow ring $\mathrm{CH}(\overline{\mathrm{OG}})_{\frac{1}{2}}$; the beginning of our analysis follows that of [Ma], [T1], [T3]. Endow the trivial bundle $E(\mathbb{C})$ over OG( $\mathbb{C})$ with a (trivial) hermitian metric $h$ compatible with the quadratic form $q$ (i.e., such that the real part of $h$ is the symmetric bilinear form on $E(\mathbb{C})$ induced by $q$ ). The metric $h$ induces metrics on the bundles $S, S^{*}$, and $\&$ becomes a sequence of hermitian vector bundles

$$
\overline{\mathcal{E}}: 0 \longrightarrow \bar{S} \longrightarrow \bar{E} \longrightarrow \bar{S}^{*} \longrightarrow 0 .
$$

The Kähler form $\omega_{\mathrm{OG}}=c_{1}\left(\bar{S}^{*}\right) / 2$ turns $\mathrm{OG}(\mathbb{C})$ into a hermitian symmetric space with compact presentation

$$
\mathrm{OG}(\mathbb{C}) \cong \mathrm{SO}(2 n+2) / \mathrm{U}(n+1)
$$

Let $\bar{G}=\left(\mathrm{OG}, \omega_{\mathrm{OG}}\right)$ denote the corresponding Arakelov variety, in the sense of [GS1].

The Chow ring $\mathrm{CH}(\mathrm{OG})$ and the ring $\operatorname{Harm}\left(\mathrm{OG}_{\mathbb{R}}\right)$ of real $\omega_{\mathrm{OG}}$-harmonic differential forms on $\mathrm{OG}(\mathbb{C})$ are related by natural isomorphisms

$$
\mathrm{CH}(\mathrm{OG}) \otimes_{\mathbb{Z}} \mathbb{R} \cong \operatorname{Harm}\left(\mathrm{OG}_{\mathbb{R}}\right) \cong \mathrm{H}^{*}(\mathrm{OG}(\mathbb{C}), \mathbb{R}),
$$

where the third ring $\mathrm{H}^{*}(\mathrm{OG}(\mathbb{C}), \mathbb{R})$ is cohomology with real coefficients.

Elements in the Arakelov Chow group $\mathrm{CH}^{p}(\overline{\mathrm{OG}})$ are represented by arithmetic cycles $\left(Z, g_{Z}\right)$, where $Z$ is a codimension $p$ cycle on OG and $g_{Z}$ is a current of type $(p-1, p-1)$ such that the current $d d^{c} g_{Z}+\delta_{Z(\mathbb{C})}$ is represented by a differential form in $\operatorname{Harm}^{p, p}\left(\mathrm{OG}_{\mathbb{R}}\right)$. Since the homogeneous space $\mathrm{OG}$ admits a natural cellular 
decomposition, it follows that for each $p$, the exact sequence of [GS1, §3.3.5] is of the form

$$
0 \longrightarrow \operatorname{Harm}^{p-1, p-1}\left(\mathrm{OG}_{\mathbb{R}}\right) \stackrel{a}{\longrightarrow} \mathrm{CH}^{p}(\overline{\mathrm{OG}}) \stackrel{\zeta}{\longrightarrow} \mathrm{CH}^{p}(\mathrm{OG}) \longrightarrow 0,
$$

where the maps $a$ and $\zeta$ are defined by

$$
a(\eta)=(0, \eta) \quad \text { and } \quad \zeta\left(Z, g_{Z}\right)=Z .
$$

Summing (3) over all $p$ gives the sequence

$$
0 \longrightarrow \operatorname{Harm}\left(\mathrm{OG}_{\mathbb{R}}\right) \stackrel{a}{\longrightarrow} \mathrm{CH}(\overline{\mathrm{OG}}) \stackrel{\zeta}{\longrightarrow} \mathrm{CH}(\mathrm{OG}) \longrightarrow 0 .
$$

For any symmetric polynomial $\phi$, we will also require the differential forms $\phi\left(\bar{S}^{*}\right)$ in $\operatorname{Harm}\left(\mathrm{OG}_{\mathbb{R}}\right)$ given by Chern-Weil theory, and the arithmetic characteristic classes $\hat{\phi}\left(\bar{S}^{*}\right)$ in $\mathrm{CH}(\overline{\mathrm{OG}})$. As in [T2], [T3], we agree that symmetric functions $\phi(\hat{x})$ and $\psi(x)$ in the formal root variables $\hat{x}=\left\{\hat{x}_{1}, \ldots, \hat{x}_{n+1}\right\}$ and $x=\left\{x_{1}, \ldots, x_{n}\right\}$ denote arithmetic classes $\hat{\phi}\left(\bar{S}^{*}\right)$ and characteristic forms $\psi\left(\bar{S}^{*}\right)$, respectively. The latter are identified, via the inclusion $a$, with elements in $\mathrm{CH}(\overline{\mathrm{OG}})$.

Consider the abelian group

$$
A=\mathbb{Z}_{\frac{1}{2}}\left[\hat{x}_{1}, \ldots, \hat{x}_{n+1}\right]^{S_{n+1}} \oplus \mathbb{R}\left[x_{1}, \ldots, x_{n}\right]^{S_{n}} .
$$

We adopt the notational convention that $\hat{\alpha}$ denotes $\hat{\alpha} \oplus 0, \beta$ denotes $0 \oplus \beta$ and any product $\prod \alpha_{i} \beta_{j}$ denotes $0 \oplus \prod \alpha_{i} \beta_{j}$, and define a product . in $A$ by imposing the relations $\hat{\alpha} \cdot \beta=\alpha \beta$ and $\beta_{1} \cdot \beta_{2}=0$. In the first of these relations, the specialization $\hat{\alpha} \mapsto \alpha$ means that we remove the 'hats' from all the variables $\hat{x}_{i}$ and set the extraneous variable $x_{n+1}$ equal to zero. Define the harmonic numbers $\mathscr{H}_{r}$ by

$$
\mathscr{H}_{r}=1+\frac{1}{2}+\cdots+\frac{1}{r}
$$

and let $p_{r}(x)=\sum x_{i}^{r}$ denote the $r$-th power sum in the variables $x_{i}$.

Consider the following two sets of relations in $A$ :

$$
\begin{gathered}
\mathcal{R}_{1}: e_{k}\left(x^{2}\right)=0, \quad 1 \leqslant k \leqslant n, \\
\mathcal{R}_{2}: e_{k}\left(\hat{x}^{2}\right)=(-1)^{k-1} \mathcal{H}_{2 k-1} p_{2 k-1}(x), \quad 1 \leqslant k \leqslant n, e_{n+1}(\hat{x})=\frac{1}{2} \mathcal{H}_{n} e_{n}(x),
\end{gathered}
$$

where $e_{k}$ denotes the $k$-th elementary symmetric function in the indicated variables. Let $\mathcal{A}$ denote the quotient of the graded ring $A$ by the relations $\mathcal{R}_{1}$ and $\mathcal{R}_{2}$.

Theorem 1. There is a unique ring isomorphism

$$
\Phi: \mathcal{A} \rightarrow \mathrm{CH}(\overline{\mathrm{OG}})_{\frac{1}{2}}
$$

such that

$$
\Phi\left(e_{k}(\hat{x})\right)=\hat{c}_{k}\left(\bar{S}^{*}\right), \quad \Phi\left(e_{k}(x)\right)=c_{k}\left(\bar{S}^{*}\right) .
$$


Proof. The initial part of the argument is similar to that in [T2, Thm. 1], so we will outline the essential points. The inclusion and projection morphisms

$$
\mathbb{R}\left[x_{1}, \ldots, x_{n}\right]^{S_{n}} \stackrel{i}{\longrightarrow} A \stackrel{\pi}{\longrightarrow} \mathbb{Z}_{\frac{1}{2}}\left[\hat{x}_{1}, \ldots, \hat{x}_{n+1}\right]^{S_{n+1}}
$$

induce an exact sequence of abelian groups:

$$
0 \longrightarrow \mathbb{R}\left[x_{1}, \ldots, x_{n}\right]^{S_{n}} /\left(\mathcal{R}_{1}\right) \stackrel{i}{\longrightarrow} \mathcal{A} \stackrel{\pi}{\longrightarrow} \mathbb{Z}_{\frac{1}{2}}\left[\hat{x}_{1}, \ldots, \hat{x}_{n+1}\right]^{S_{n+1}} /\left(\widehat{\mathcal{R}}_{2}\right) \longrightarrow 0
$$

where the relations $\widehat{\mathcal{R}}_{2}$ are defined by

$$
\widehat{\mathcal{R}}_{2}: e_{k}\left(\hat{x}^{2}\right)=0, \quad 1 \leqslant k \leqslant n, e_{n+1}(\hat{x})=0 .
$$

To show that $\Phi$ is an isomorphism one uses the isomorphisms (2), the relations (i), (ii) in $\mathrm{CH}(\mathrm{OG})_{\frac{1}{2}}$, and the five lemma to identify the short exact sequences (4) and (5) (as in loc. cit.). Our definition of the product - agrees with the $\mathrm{CH}(\bar{G})$-module structure of the square zero ideal $\operatorname{Harm}\left(G_{\mathbb{R}}\right) \hookrightarrow \mathrm{CH}(\bar{G})$ (see [GS1]).

The first set of $n$ relations in $\mathcal{R}_{2}$ come from the equation

$$
\hat{c}(\bar{S}) \cdot \hat{c}\left(\bar{S}^{*}\right)=1+\tilde{c}(\overline{\mathcal{E}}) .
$$

Here $\tilde{c}(\overline{\mathcal{E}})$ is the image in $\mathrm{CH}(\bar{G})$ of the Bott-Chern form of the exact sequence $\overline{\mathcal{E}}$ for the total Chern class (see [BC] and [GS2]). According to [T1, Prop. 3], we have

$$
\tilde{c}_{j}(\overline{\mathcal{E}})=(-1)^{j-1} \mathscr{H}_{j-1} p_{j-1}\left(\bar{S}^{*}\right)
$$

for all $j$; note that $\tilde{c}_{j}(\overline{\mathcal{E}})$ vanishes when $j$ is odd. If we express the two previous equations using root notation we obtain

$$
e_{k}\left(\hat{x}_{1}^{2}, \ldots, \hat{x}_{n+1}^{2}\right)=(-1)^{k-1} \mathscr{H}_{2 k-1} p_{2 k-1}\left(x_{1}, \ldots, x_{n}\right)
$$

for $1 \leqslant k \leqslant n$. These are the first set of relations in $\mathcal{R}_{2}$.

The last relation in $\mathcal{R}_{2}$ is new, and is equivalent to the equation

$$
\hat{c}_{n+1}\left(\bar{S}^{*}\right)=\frac{1}{2} \mathscr{H}_{n} c_{n}\left(\bar{S}^{*}\right)
$$

We first claim that there is a constant $r_{n}$ such that

$$
\hat{c}_{n+1}\left(\bar{S}^{*}\right)=r_{n} c_{n}\left(\bar{S}^{*}\right) .
$$

To see this, observe that $\hat{c}_{n+1}\left(\bar{S}^{*}\right)$ lies in $\operatorname{Ker}(\zeta)$, and hence is the image (under $a$ ) of a linear combination of harmonic forms of degree $2 n$ on OG $(\mathbb{C})$. These harmonic forms 
are Poincaré dual to Schubert classes on OG; hence $\hat{c}_{n+1}\left(\bar{S}^{*}\right)$ is a linear combination of Schubert forms $\tau_{\lambda}=\tau_{\lambda}\left(\bar{S}^{*}\right)$ (see $\$ 3.2$ ).

Next, consider the natural inclusion $j: \mathrm{OG}(n, 2 n) \hookrightarrow \mathrm{OG}(n+1,2 n+2)$ obtained by writing the vector space $E=k^{2 n} \oplus k^{2}$. The bundle $j^{*} \bar{S}$ splits as an orthogonal direct sum $\bar{S}_{n} \oplus \bar{L}$, where $\bar{S}_{n}$ is the tautological hermitian vector bundle over $\operatorname{OG}(n, 2 n)$ and $\bar{L}$ is a trivial hermitian line bundle. It follows that $j^{*} \hat{c}_{n+1}\left(\bar{S}^{*}\right)=0$. The point now is that each Schubert form $\tau_{\lambda}$ of degree $2 n$ on OG $(n+1,2 n+2)$ restricts to a corresponding (non-zero) Schubert form $\tau_{\lambda}$ on $\mathrm{OG}(n, 2 n)$, with the exception of the special Schubert form $c_{n}\left(\bar{S}^{*}\right)$, which vanishes when restricted to $\operatorname{OG}(n, 2 n)$. This proves the above claim (7). More work is required to obtain the precise value of the constant $r_{n}$; we will do this after we study arithmetic Schubert calculus on OG.

Remark. As in [T3, §2], the relations $\mathcal{R}_{1}$ and $\mathcal{R}_{2}$ may be expressed in the form

$$
\begin{gathered}
\mathcal{R}_{1}^{\prime}: \prod_{i=1}^{n}\left(1-x_{i}^{2} t^{2}\right)=1, \\
\mathcal{R}_{2}^{\prime}: \prod_{j=1}^{n+1}\left(1-\hat{x}_{j}^{2} t^{2}\right) \cdot\left(1+q_{a}(x, t)\right)=1, \quad \prod_{j=1}^{n+1} \hat{x}_{j}=\frac{1}{2} \mathscr{H}_{n} \prod_{i=1}^{n} x_{i}
\end{gathered}
$$

where $t$ is a formal variable (note that $\mathcal{R}_{2}^{\prime}$ uses the multiplication in $A$ ). Here

$$
q_{a}(x, t)=\frac{t}{2} \sum_{i=1}^{n}\left(\frac{\log \left(1+x_{i} t\right)}{1+x_{i} t}-\frac{\log \left(1-x_{i} t\right)}{1-x_{i} t}\right) .
$$

We next give a presentation of $\mathrm{CH}(\mathrm{OG})$ and $\mathrm{CH}(\overline{\mathrm{OG}})$ with integer coefficients. For this, we will use the special Schubert classes $\tau_{i}$ and $\hat{\tau}_{i}$, defined by

$$
\tau_{k}=\frac{1}{2} c_{k}\left(S^{*}\right), 1 \leqslant k \leqslant n, \quad \text { and } \quad \hat{\tau}_{k}=\frac{1}{2} \hat{c}_{k}\left(\bar{S}^{*}\right), 1 \leqslant k \leqslant n+1 .
$$

The Chow ring $\mathrm{CH}(\mathrm{OG})$ is a quotient of the polynomial ring $\mathbb{Z}\left[\tau_{1}, \ldots, \tau_{n}\right]$ modulo the relations

$$
\tau_{k}^{2}+2 \sum_{p=1}^{k-1}(-1)^{p} \tau_{k+p} \tau_{k-p}+(-1)^{k} \tau_{2 k}=0
$$

for all $1 \leqslant k \leqslant n$ (this presentation for the cohomology ring may be derived e.g. from $[\mathrm{P}, \S 6])$. To obtain the analogous presentation of $\mathrm{CH}(\overline{\mathrm{OG}})$, as above, we identify the $\tau_{k}$ 's with the images under the map $a$ of the special Schubert forms in $\mathrm{CH}(\overline{\mathrm{OG}})$. 
Theorem 2. The ring $\mathrm{CH}(\overline{\mathrm{OG}})$ is presented as a quotient of the polynomial ring $\mathbb{Z}\left[\hat{\tau}_{1}, \ldots, \hat{\tau}_{n+1}\right] \oplus \mathbb{R}\left[\tau_{1}, \ldots, \tau_{n}\right]$ (with the aforementioned product) modulo the relations

$$
\tau_{k}^{2}+2 \sum_{p=1}^{k-1}(-1)^{p} \tau_{k+p} \tau_{k-p}+(-1)^{k} \tau_{2 k}=0
$$

for all $k \leqslant n$, together with the arithmetic relations

$$
\hat{\tau}_{k}^{2}+2 \sum_{p=1}^{k-1}(-1)^{p} \hat{\tau}_{k+p} \hat{\tau}_{k-p}+(-1)^{k} \hat{\tau}_{2 k}=-\frac{1}{2} \mathscr{H}_{2 k-1} \operatorname{det}\left(\tau_{i j}^{\prime}\right)_{1 \leqslant i, j \leqslant 2 k-1}
$$

and

$$
\hat{\tau}_{n+1}=\frac{1}{2} \mathscr{H}_{n} \tau_{n} .
$$

Here $\left\{\tau_{i j}^{\prime}\right\}$ is a matrix with $\tau_{1 j}^{\prime}=j \tau_{1+j-i}$ and $\tau_{i j}^{\prime}=\tau_{1+j-i}$ for $i>1$.

Proof. The left hand side of equation (8) is related to $e_{k}\left(\hat{x}^{2}\right)$ by the formula

$$
\left(1+2 \sum_{k=1}^{n+1} \hat{\tau}_{k} \eta^{k}\right) \cdot\left(1+2 \sum_{k=1}^{n+1}(-1)^{k} \hat{\tau}_{k} \eta^{k}\right)=\prod_{j=1}^{n+1}\left(1-\hat{x}_{j}^{2} \eta^{2}\right),
$$

where $\eta$ is a formal variable. Expanding both sides of (9) and equating terms of like degree gives

$$
\hat{\tau}_{k}^{2}+2 \sum_{p=1}^{k-1}(-1)^{p} \hat{\tau}_{k+p} \hat{\tau}_{k-p}+(-1)^{k} \hat{\tau}_{2 k}=\frac{(-1)^{k}}{2} e_{k}\left(\hat{x}^{2}\right) .
$$

Relation $\mathcal{R}_{2}$ equates the right hand side of (10) with $-\frac{1}{2} \mathscr{H}_{2 k-1} p_{2 k-1}(x)$. We now use the formula from [M, Ex. I.2.8] which expresses the power sums as a polynomial in the elementary symmetric functions. This gives

$$
p_{2 k-1}(x)=\operatorname{det}\left(\tau_{i j}^{\prime}\right)_{1 \leqslant i, j \leqslant 2 k-1} .
$$

The rest of the relations in the theorem are clear.

\section{Arithmetic Schubert calculus}

3.1. $\tilde{\boldsymbol{P}}$-polynomials. We will require the basic facts about partitions and their Young diagrams which were used in [T3]. A partition $\lambda=\left(\lambda_{1}, \ldots, \lambda_{r}\right)$ with distinct nonzero parts $\lambda_{i}$ is called strict. The length $\ell(\lambda)$ is the number of non-zero parts $\lambda_{i}$, 
and the weight $|\lambda|=\sum \lambda_{i}$. Each partition $\lambda$ is identified with its Young diagram of boxes, and this is used to define the containment relation $\lambda \supset \mu$ for partitions. We let $D_{n}$ denote the set of strict partitions $\lambda$ with $\lambda_{1} \leqslant n$. The shifted diagram of a strict partition $\lambda$, denoted $\delta(\lambda)$, is obtained by shifting the $i$-th row of the diagram of $\lambda$ $i-1$ squares to the right, for each $i>1$ (see Figure 1). For $\lambda \supset \mu$, the shifted skew diagram $\delta(\lambda / \mu)$ is obtained by removing the boxes in $\delta(\mu)$ from those of $\delta(\lambda)$.
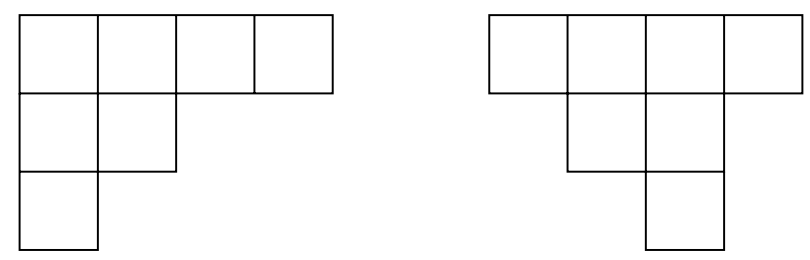

Figure 1. $\lambda$ and $\&(\lambda)$ for $\lambda=(4,2,1)$.

The $\widetilde{P}$-polynomials of Pragacz and Ratajski $[\mathrm{PR}]$ will be useful in our description of Schubert calculus on OG. Let $X=\left(X_{1}, \ldots, X_{n}\right)$ be an $n$-tuple of variables and define $\widetilde{P}_{0}(X)=1$ and $\widetilde{P}_{i}(X)=e_{i}(X) / 2$ for each $i>0$. For nonnegative integers $i, j$ with $i \geqslant j$, set

$$
\widetilde{P}_{i, j}(X)=\widetilde{P}_{i}(X) \widetilde{P}_{j}(X)+2 \sum_{k=1}^{j-1}(-1)^{k} \widetilde{P}_{i+k}(X) \widetilde{P}_{j-k}(X)+(-1)^{j} \widetilde{P}_{i+j}(X),
$$

and for any partition $\lambda$ of length $\ell=\ell(\lambda)$, not necessarily strict, define

$$
\widetilde{P}_{\lambda}(X)=\operatorname{Pfaffian}\left[\widetilde{P}_{\lambda_{i}, \lambda_{j}}(X)\right]_{1 \leqslant i<j \leqslant r},
$$

where $r$ is the smallest even integer such that $r \geqslant \ell(\lambda)$.

These polynomials are related to the $\widetilde{Q}$-polynomials used in [T3] by the equation

$$
\widetilde{P}_{\lambda}(X)=2^{-\ell(\lambda)} \widetilde{Q}_{\lambda}(X) .
$$

If $\Lambda_{n}^{\prime}$ denotes the $\mathbb{Z}$-algebra generated by the polynomials $\widetilde{P}_{\lambda}(X)$ for all $\lambda \in \mathscr{D}_{n}$, then $\Lambda_{n}^{\prime}$ is isomorphic to the ring $\Lambda_{n}=\mathbb{Z}[X]^{S_{n}}$ of symmetric polynomials in $X$, and the set $\left\{\widetilde{P}_{\lambda}(X) \mid \lambda_{1} \leqslant n\right\}$ is a free $\mathbb{Z}$-basis of $\Lambda_{n}^{\prime}$. It follows that there exist integers $f_{\lambda \mu}^{v}$, independent of $n$, such that

$$
\widetilde{P}_{\lambda}(X) \widetilde{P}_{\mu}(X)=\sum_{\nu} f_{\lambda \mu}^{v} \widetilde{P}_{\nu}(X) .
$$

The corresponding coefficients $e_{\lambda \mu}^{\nu}$ in the expansion of the product $\widetilde{Q}_{\lambda}(X) \widetilde{Q}_{\mu}(X)$ are related to the $f_{\lambda \mu}^{\nu}$ by the equation

$$
e_{\lambda \mu}^{\nu}=2^{\ell(\lambda)+\ell(\mu)-\ell(\nu)} f_{\lambda \mu}^{v}
$$


There are explicit combinatorial rules (involving signs in general) for computing the integers $f_{\lambda \mu}^{\nu}$, which follow from corresponding formulas for decomposing products of Hall-Littlewood polynomials. When $\lambda, \mu$ and $v$ are strict partitions, the $f_{\lambda \mu}^{v}$ are classical Schubert structure constants for $\mathrm{OG}(n+1,2 n+2)$, and hence nonnegative.

Recall that a skew Young diagram $\mu / \lambda$ is a horizontal strip if it has at most one box in each column. Define the connected components of such a diagram by specifying that two boxes are connected if they share a vertex or an edge. We then have the following Pieri type formula for $\lambda$ strict:

$$
\widetilde{P}_{\lambda}(X) \widetilde{P}_{k}(X)=\sum_{\mu} 2^{N(\lambda, \mu)} \widetilde{P}_{\mu}(X),
$$

where the sum is over all partitions $\mu \supset \lambda$ with $|\mu|=|\lambda|+k$ such that $\mu / \lambda$ is a horizontal strip, and $N(\lambda, \mu)$ is one less than the number of connected components of $\mu / \lambda$. In particular, we have

$$
\widetilde{P}_{\lambda}(X) \widetilde{P}_{n}(X)=\widetilde{P}_{(n, \lambda)}(X)
$$

for all $\lambda \in D_{n}$.

In [T3, Prop. 1] we proved a combinatorial formula for the product $\widetilde{Q}_{1}(X)^{N}$, which implies that

$$
\widetilde{P}_{1}(X)^{N}=\sum_{|\lambda|=N} g^{\lambda} \widetilde{P}_{\lambda}(X) .
$$

Here $g^{\lambda}$ denotes the number of proper standard tableaux of shape $\lambda$, in the sense of $[\mathrm{T} 3, \S 3]$. We say that a standard tableau $T$ on $\lambda$ is proper if for each position $(i . j)$ of a box in $\lambda$, there is an odd number of entries of $T$ which (i) lie in positions $(k, j)$ for some $k \geqslant i$ and (ii) are less than the $(i, j+1)$ entry (the condition is vacuous if $\lambda$ has no box in the $(i, j+1)$ position). In case $\lambda$ is strict, $g^{\lambda}$ counts the number of standard tableaux of shape $\delta(\lambda)$, and is given by an explicit formula due to Schur [S] (see also [M, Ex. III.8.12]):

$$
g^{\lambda}=\frac{|\lambda| !}{\prod_{i} \lambda_{i} !} \cdot \frac{\prod_{i<j}\left(\lambda_{i}-\lambda_{j}\right)}{\prod_{i<j}\left(\lambda_{i}+\lambda_{j}\right)} .
$$

3.2. Classical theory. We review here the classical Schubert calculus which describes the multiplicative structure of $\mathrm{CH}(\mathrm{OG})$ with respect to the basis of Schubert classes, following $[\mathrm{P}, \S 6]$. We agree that $\tau_{\lambda}(\mathrm{x})$ will denote $\widetilde{P}_{\lambda}(\mathrm{x})$, where $\mathrm{x}=\left\{\mathrm{x}_{1}, \ldots, \mathrm{x}_{n}\right\}$ are the Chern roots of the vector bundle $S^{*}$ (we have set the last root variable $\mathrm{x}_{n+1}=0$ here). Similar conventions are used when dealing with $\widetilde{P}$. polynomials in the other two kinds of root variables discussed in $\$ 2$. 
The abelian group $\mathrm{CH}(\mathrm{OG})$ is freely generated by the Schubert classes $\tau_{\lambda}(\mathrm{x})=$ $\tau_{\lambda}\left(S^{*}\right)$, for all strict partitions $\lambda$ in $D_{n}$. Recall that $\tau_{\lambda}(\mathrm{x})$ is the class of the codimension $|\lambda|$ Schubert variety $X_{\lambda}$, defined as follows: if $\left\{e_{i}\right\}$ is the basis of $E$ chosen in $\S 2$ and $F_{k}=\operatorname{Span}\left\langle e_{1}, \ldots, e_{k}\right\rangle$ then $X_{\lambda}$ parametrizes the set

$$
\left\{V \in \mathrm{OG}(k) \mid \operatorname{dim}\left(V \cap F_{n+1-\lambda_{i}}\right) \geqslant i \text { for } 1 \leqslant i \leqslant \ell(\lambda)\right\}
$$

over any base field $k$.

The formulas in $\$ 3.1$ give the following multiplication rules in $\mathrm{CH}(\mathrm{OG})$ : for any two partitions $\lambda, \mu \in D_{n}$,

$$
\tau_{\lambda}(\mathrm{x}) \tau_{\mu}(\mathrm{x})=\sum_{\nu \in D_{n}} f_{\lambda \mu}^{v} \tau_{\nu}(\mathrm{x})
$$

the non-negative integers $f_{\lambda \mu}^{v}$ are the structure constants in $\mathrm{CH}(\mathrm{OG})$. When $\mu=k$ is a single integer then $\tau_{\mu}(\mathrm{x})=\tau_{k}(\mathrm{x})$ is a special Schubert class, and (17) specializes to the following Pieri rule (due to Hiller and Boe [HB]):

$$
\tau_{\lambda}(\mathrm{x}) \tau_{k}(\mathrm{x})=\sum 2^{N(\lambda, \mu)} \tau_{\mu}(\mathrm{x})
$$

the sum over all (strict) partitions $\mu \supset \lambda$ with $|\mu|=|\lambda|+k$ such that $\mu / \lambda$ is a horizontal strip, with $N(\lambda, \mu)$ defined as in $\S 3.1$. Since $\operatorname{OG}(\mathbb{C})$ is a hermitian symmetric space, (17) and (18) are valid on the level of harmonic differential forms on $\mathrm{OG}(\mathbb{C})$.

3.3. Schubert calculus in $\mathbf{C H}(\overline{\mathbf{O G}})$. We are now ready to extend the classical Schubert calculus described in $\S 3.2$ to $\mathrm{CH}(\overline{\mathrm{OG}})$. An edge-connected skew diagram $\gamma$ is called a rim hook if it contains no $2 \times 2$ square; the height ht $(\gamma)$ of $\gamma$ is one less than the number of rows it occupies. We define, in the context of shifted diagrams, a double rim to be the skew diagram formed by the union of two rim hooks which both end on the main diagonal $\Delta=\{(i, i) \mid i>0\}$. Each double $\operatorname{rim} \delta=\alpha \cup \beta$ is a union of two non-empty edge-connected pieces; $\alpha$ consists of the diagonals of length two in $\delta$ (which are parallel to $\Delta$ ) and $\beta=\delta \backslash \alpha$ is a rim hook. In this case we say that the double rim is of type $\left(\frac{1}{2}|\alpha|,|\beta|\right)$; a double rim of type $(2,3)$ appears in Figure 2. For any such double rim $\delta$ and for any single rim hook $\gamma$, let

$$
\varepsilon(\delta)=(-1)^{|\alpha| / 2+\mathrm{ht}(\beta)} 2 \text { and } \varepsilon(\gamma)=(-1)^{\mathrm{ht}(\gamma)} .
$$

Define $\rho(n)$ to be the partition $(n, n-1, \ldots, 1)$, so that $D_{n}$ consists of those strict partitions $\lambda$ with $\lambda \subset \rho(n)$. Following [T3, §4.2], we let $E_{n}$ be the set of non-strict partitions $\lambda$ with $\lambda_{1} \leqslant n$ such that exactly one non-zero part $r_{\lambda}$ of $\lambda$ occurs more than once, and further, $r_{\lambda}$ occurs 2 or 3 times. In addition, let $E_{n}^{\prime}$ be the set of strict partitions of the form $\left(n+1, \lambda^{\prime}\right)$, where $\lambda^{\prime} \subset \rho(n-1)$. Define a map

$$
E_{n} \cup E_{n}^{\prime} \longrightarrow D_{n}: \quad \lambda \longmapsto \bar{\lambda}
$$


as follows: if $\lambda \in E_{n}$, let $\bar{\lambda}$ be $\lambda$ minus two of the repeated parts $r_{\lambda}$, and if $\lambda=$ $\left(n+1, \lambda^{\prime}\right)$, let $\bar{\lambda}=\left(n, \lambda^{\prime}\right)$. For example, if $n=6, \lambda=(6,5,5,5,2)$, and $\mu=$ $(7,5,2)$, then $\bar{\lambda}=\bar{\mu}=(6,5,2)$.

Suppose that $\lambda \in E_{n}$ and $\mu \in D_{n}$ are two Young diagrams with $|\mu|=|\lambda|-1$. We say that there is a shifted hook operation from $\lambda$ to $\mu$ if the shifted skew diagram $\delta(\mu / \bar{\lambda})$ is a rim hook or double rim (with $2 r_{\lambda}-1$ boxes). If $\lambda \in E_{n}^{\prime}$, we say that there is a row operation from $\lambda$ to $\mu$ if $\mu=\bar{\lambda}$. A mixed operation from $\lambda \in E_{n} \cup E_{n}^{\prime}$ to $\mu \in D_{n}$ is a shifted hook operation (if $\lambda \in E_{n}$ ) or a row operation (if $\lambda \in E_{n}^{\prime}$ ) from $\lambda$ to $\mu$. Figure 2 illustrates three mixed operations to the partition $(5,3,2)$, when $n=5$.
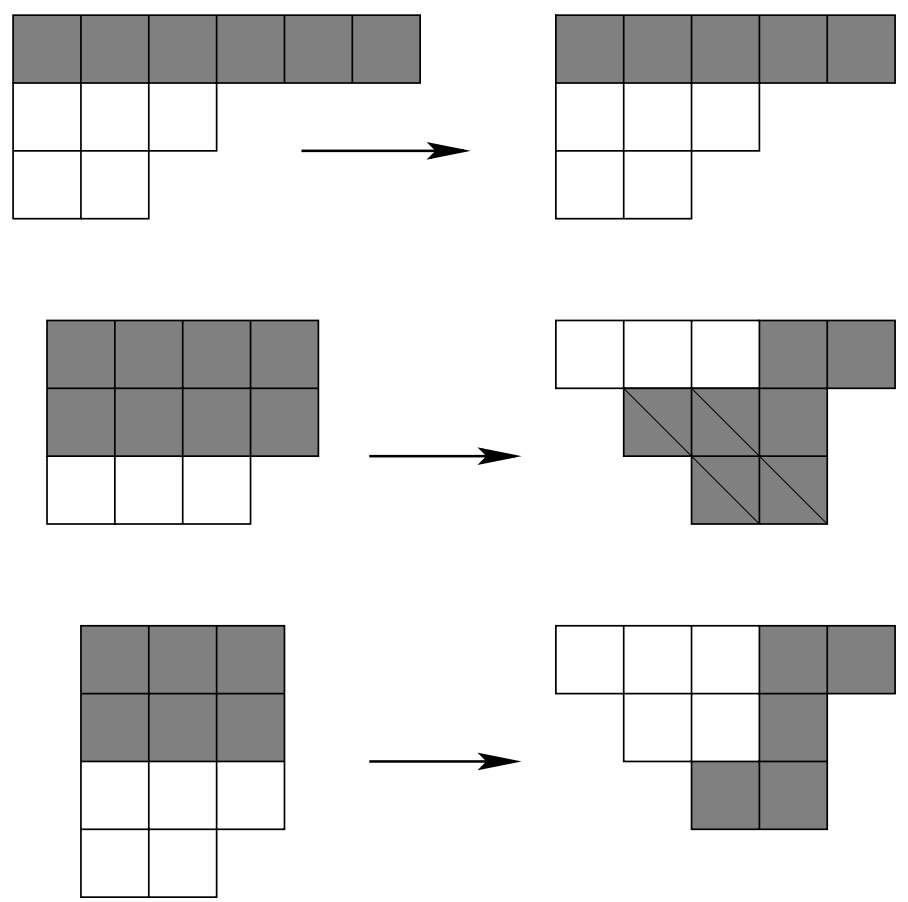

Figure 2. One row and two shifted hook operations to $(5,3,2)$.

It is clear that there is at most one mixed operation from $\lambda$ to $\mu$; it determines an integer $\varepsilon_{\lambda \mu} \in\{ \pm 1, \pm 2\}$ defined by

$$
\varepsilon_{\lambda \mu}= \begin{cases}(-1)^{r_{\lambda}-1} \varepsilon(8(\mu / \bar{\lambda})) & \text { if } \lambda \in E_{n}, \\ 1 & \text { if } \lambda \in E_{n}^{\prime}\end{cases}
$$


and a rational number $\vartheta_{\lambda}^{\mu}$ by

$$
\vartheta_{\lambda}^{\mu}= \begin{cases}\frac{1}{2} \varepsilon_{\lambda \mu} \mathscr{H}_{2 r_{\lambda}-1} & \text { if } \lambda \in E_{n}, \\ \frac{1}{2} \mathscr{H}_{n} & \text { if } \lambda \in E_{n}^{\prime} .\end{cases}
$$

If there is no mixed operation from $\lambda$ to $\mu$ then set $\vartheta_{\lambda}^{\mu}=0$.

Next we define the arithmetic structure constants $\tilde{f}_{\lambda \mu}^{v}$ : for any $\nu \in E_{n}$ and $\lambda, \mu$ strict such that $|\nu|=|\lambda|+|\mu|-1$ let

$$
\tilde{f}_{\lambda \mu}^{\nu}=\sum_{\rho \in E_{n} \cup E_{n}^{\prime}} \vartheta_{\rho}^{\nu} f_{\lambda \mu}^{\rho}
$$

where the $f_{\lambda \mu}^{\rho}$ are defined by (12). Observe that only partitions $\rho$ such that there is a mixed operation from $\rho$ to $v$ contribute to the sum (19).

Theorem 3. (a) Let $p$ be an integer between 0 and $\left(\begin{array}{c}n+1 \\ 2\end{array}\right)+1$. Each element $z \in$ $\mathrm{CH}^{p}(\overline{\mathrm{OG}})$ has a unique expression

$$
z=\sum_{\substack{\lambda \in D_{n} \\|\lambda|=p}} c_{\lambda} \tau_{\lambda}(\hat{x})+\sum_{\substack{\lambda \in D_{n} \\|\lambda|=p-1}} \gamma_{\lambda} \tau_{\lambda}(x),
$$

where $c_{\lambda} \in \mathbb{Z}$ and $\gamma_{\lambda} \in \mathbb{R}$.

(b) For $\lambda$ and $\mu$ in $D_{n}$ we have the multiplication rules

$$
\begin{gathered}
\tau_{\lambda}(\hat{x}) \cdot \tau_{\mu}(\hat{x})=\sum_{\substack{\nu \in D_{n} \\
|\nu|=|\lambda|+|\mu|}} f_{\lambda \mu}^{v} \tau_{\nu}(\hat{x})+\sum_{\substack{v \in D_{n} \\
|v|=|\lambda|+|\mu|-1}} \tilde{f}_{\lambda \mu}^{v} \tau_{\nu}(x), \\
\tau_{\lambda}(\hat{x}) \cdot \tau_{\mu}(x)=\sum_{\substack{\nu \in D_{n} \\
|\nu|=|\lambda|+|\mu|}} f_{\lambda \mu}^{v} \tau_{\nu}(x), \\
\tau_{\lambda}(x) \cdot \tau_{\mu}(x)=0 .
\end{gathered}
$$

Proof. The argument is similar to the proof of [T3, Thm. 2], and we will discuss the main points here. First, we use the morphism $\varepsilon: \mathrm{CH}(\mathrm{OG}) \rightarrow \mathrm{CH}(\overline{\mathrm{OG}})$ defined by $\varepsilon\left(\tau_{\lambda}(\mathrm{x})\right)=\tau_{\lambda}(\hat{x})$ (for each $\lambda \in D_{n}$ ) to split the exact sequence (4). We thus obtain an isomorphism of abelian groups

$$
\mathrm{CH}(\overline{\mathrm{OG}}) \cong \mathrm{CH}(\mathrm{OG}) \oplus \operatorname{Harm}\left(\mathrm{OG}_{\mathbb{R}}\right),
$$

proving the statement in part (a). By the definition, the $\tau_{\lambda}(\hat{x})$ for $\lambda \in D_{n}$ are the arithmetic Schubert classes. 
The only difficulty in part (b) lies in proving the first equality. We use the fact that the arithmetic characteristic class map is an algebra homomorphism; this gives the identity

$$
\tau_{\lambda}(\hat{x}) \cdot \tau_{\mu}(\hat{x})=\sum_{\substack{\nu \in D_{n} \\|\nu|=|\lambda|+|\mu|}} f_{\lambda \mu}^{v} \tau_{\nu}(\hat{x})+\sum_{\substack{\rho \in E_{n} \cup E_{n}^{\prime} \\|\rho|=|\lambda|+|\mu|}} f_{\lambda \mu}^{\rho} \tau_{\rho}(\hat{x}) .
$$

All of the classes $\tau_{\rho}(\hat{x})$ which appear in the second sum in (20) lie in the image of $a: \operatorname{Harm}\left(\mathrm{OG}_{\mathbb{R}}\right) \hookrightarrow \mathrm{CH}(\overline{\mathrm{OG}})$, and it remains to equate them with explicit harmonic forms on $\mathrm{OG}(\mathbb{C})$. This is done in the following result.

Proposition 1. For partitions $\lambda \in E_{n} \cup E_{n}^{\prime}$, we have

$$
\tau_{\lambda}(\hat{x})=\sum_{\nu} \vartheta_{\lambda}^{\nu} \tau_{\nu}(x)
$$

the sum over all $v \in D_{n}$ that can be obtained from $\lambda$ by a mixed operation. If $\lambda \notin D_{n} \cup E_{n} \cup E_{n}^{\prime}$ then $\tau_{\lambda}(\hat{x})=0$.

Proof. For $\lambda \in E_{n}$, the relation (11) between $\widetilde{P}$ - and $\widetilde{Q}$-polynomials and [T3, Eq. (18)] give

$$
\tau_{\lambda}(\hat{x})=\frac{1}{4} \tau_{\bar{\lambda}}(\hat{x}) \cdot e_{r_{\lambda}}\left(\hat{x}^{2}\right)=\frac{(-1)^{r_{\lambda}-1}}{4} \mathscr{H}_{2 r_{\lambda}-1} p_{2 r_{\lambda}-1}(x) \tau_{\bar{\lambda}}(x) ;
$$

here we have used the first set of relations in $\mathcal{R}_{2}$ of $\S 2$. The following rule for multiplying a $\widetilde{P}$-polynomial by a power sum $p_{r}(x)$ with $r$ odd in $\operatorname{Harm}\left(\mathrm{OG}_{\mathbb{R}}\right)$ is derived from [T3, Eq. (19)]:

$$
p_{r}(x) \tau_{\mu}(x)=2 \sum_{\nu} \varepsilon(\delta(v / \mu)) \tau_{v}(x),
$$

the sum over all strict $\nu \supset \mu$ with $|\nu|=|\mu|+r$ such that $\delta(\nu / \mu)$ is a rim hook or a double rim. One now combines (22) with (23) to prove (21) in the case when $\lambda \in E_{n}$.

If $\lambda=\left(n+1, \lambda^{\prime}\right) \in E_{n}^{\prime}$, we use equation (14) twice and the last relation in $\mathcal{R}_{2}$ to obtain

$$
\tau_{\lambda}(\hat{x})=\tau_{n+1}(\hat{x}) \cdot \tau_{\lambda^{\prime}}(\hat{x})=\frac{1}{2} \mathscr{H}_{n} \tau_{n}(x) \cdot \tau_{\lambda^{\prime}}(\hat{x})=\frac{1}{2} \mathscr{H}_{n} \tau_{\bar{\lambda}}(x) .
$$

Finally, the fact that $\operatorname{Im}(a)$ is a square zero ideal in $\mathrm{CH}(\overline{\mathrm{OG}})$ implies that for all partitions $\lambda$ with $\lambda \notin D_{n} \cup E_{n} \cup E_{n}^{\prime}$, we have $\tau_{\lambda}(\hat{x})=0$. This completes the proof of the proposition.

To finish the proof of Theorem 3, substitute (21) in the second sum in (20) and gather like terms. 
The Pieri formula (13) is used to obtain the following special case of Theorem 3:

Corollary 1 (Arithmetic Pieri rule). Let $C(\lambda, k)$ be the set of partitions $\mu \supset \lambda$ with $|\mu|=|\lambda|+k$ such that $\mu / \lambda$ is a horizontal strip. Then for $\lambda \in D_{n}$ we have

$$
\tau_{\lambda}(\hat{x}) \cdot \tau_{k}(\hat{x})=\sum_{\mu} 2^{N(\lambda, \mu)} \tau_{\mu}(\hat{x})+\sum_{\nu}\left(\sum_{\rho} 2^{N(\lambda, \rho)} \vartheta_{\rho}^{\nu}\right) \tau_{\nu}(x),
$$

where the first (classical) sum is over $\mu \in D_{n} \cap C(\lambda, k)$ and the second sum is over $v$ and $\rho$ with $\rho \in\left(E_{n} \cup E_{n}^{\prime}\right) \cap C(\lambda, k)$.

\section{The Faltings height of $O G$}

In this section, we use arithmetic Schubert calculus to compute the Faltings height of OG with respect to its fundamental embedding in projective space. This is the embedding given by the generator $L$ of $\operatorname{Pic}(\mathrm{OG})$ with $c_{1}(L)=\tau_{1}\left(S^{*}\right)$. In geometry the degree of $\mathrm{OG}(k)$ (for any field $k$ ) with respect to $L=\mathcal{O}(1)$ is given by

$$
\operatorname{deg}_{\mathcal{O}(1)}(\mathrm{OG}(k))=g^{\rho(n)} \text {. }
$$

This is a direct consequence of equation (15). The Faltings height $[\mathrm{F}]$ of OG under its fundamental embedding is an arithmetic analogue of the geometric degree (24).

The natural invariant metric on projective space induces a hermitian metric on $L$. The height of $G$ with respect to $\bar{L}=\overline{\mathcal{O}}(1)$ is the number

$$
\mathrm{ht}_{\overline{\mathcal{O}}(1)}(\mathrm{OG})=\widehat{\operatorname{deg}}\left(\hat{c}_{1}(\overline{\mathcal{O}}(1))^{d} \mid \mathrm{OG}\right)=\widehat{\operatorname{deg}}\left(\tau_{1}^{d}(\hat{x})\right) .
$$

Here the arithmetic degree map $\widehat{\operatorname{deg}}$ is defined as in [BGS] and $d=\left(\begin{array}{c}n+1 \\ 2\end{array}\right)+1$ is the absolute dimension of OG. We have an equation

$$
\tau_{1}^{d}(\hat{x})=w_{n} \tau_{\rho(n)}(x)=w_{n} \tau_{\rho(n)}\left(\bar{S}^{*}\right)
$$

in $\mathrm{CH}(\overline{\mathrm{OG}})$, for some rational number $w_{n}$. The height (25) is then given by

$$
\mathrm{ht}_{\overline{\mathcal{O}(1)}}(\mathrm{OG})=\frac{1}{2} \int_{\mathrm{OG}(\mathbb{C})} w_{n} \tau_{\rho(n)}\left(\bar{S}^{*}\right)=\frac{w_{n}}{2} .
$$

The last equality holds because $\tau_{\rho(n)}\left(\bar{S}^{*}\right)$ is dual to the class of a point on $\operatorname{OG}(\mathbb{C})$.

Theorem 4. The height of the orthogonal Grassmannian $G$ with respect to $\overline{\mathcal{O}}(1)$ is given by

$$
\mathrm{ht}_{\overline{\mathcal{O}}(1)}(\mathrm{OG})=\frac{1}{2} \sum_{\substack{0 \leqslant a+2 b<n \\ a, b \geqslant 0}}(-1)^{b} 2^{-\delta_{a 0}} \mathscr{H}_{2 a+2 b+1} g^{[a, b]_{n}}+\frac{1}{4} \mathscr{H}_{n} g^{(n+1, \rho(n-1))}
$$

where $\delta_{i j}$ is the Kronecker delta. 
Proof. We recall the fundamental set of diagrams $\mathcal{E}(n)$ from [T3, §5]. First, we agree that a single rim hook $\beta$ which ends on the main diagonal of a shifted diagram is a double rim of type $(0,|\beta|)$. Then

$$
\mathcal{E}(n)=\left\{[a, b]_{n} \mid a, b \geqslant 0,0 \leqslant a+2 b<n\right\}
$$

where $[a, b]_{n}$ denotes the unique diagram $\lambda \in E_{n}$ of weight $d$ such that $\delta(\rho(n) / \bar{\lambda})$ is a double rim of type $(a, 2 b+1)$. For example, one has

$$
\begin{aligned}
\mathscr{E}(4)= & \left\{[0,0]_{4},[1,0]_{4},[2,0]_{4},[3,0]_{4},[0,1]_{4},[1,1]_{4}\right\} \\
= & \{(4,3,2,1,1),(4,3,2,2),(4,3,3,1), \\
& (4,4,2,1),(4,2,2,2,1),(3,3,3,2)\} .
\end{aligned}
$$

The partitions in $\mathcal{E}(n)$ are exactly those which admit a shifted hook operation to $\rho(n)$. Moreover, $(n+1, \rho(n-1))$ is the unique partition with a row operation to $\rho(n)$. We now use equation (15) and Proposition 1 to obtain

$$
\begin{aligned}
\tau_{1}(\hat{x})^{d} & =\sum_{\lambda \in \mathcal{E}(n)} g^{\lambda} \tau_{\lambda}(\hat{x})+g^{(n+1, \rho(n-1))} \tau_{(n+1, \rho(n-1))}(\hat{x}) \\
& =\sum_{\lambda \in \mathcal{E}(n)} g^{\lambda} \vartheta_{\lambda}^{\rho(n)} \tau_{\rho(n)}(x)+\frac{1}{2} \mathscr{H}_{n} g^{(n+1, \rho(n-1))} \tau_{\rho(n)}(x) .
\end{aligned}
$$

The sum in the last equation is evaluated exactly as in the proof of [T3, Thm. 3], and we obtain formula (26).

It is clear from equation (26) that $\mathrm{ht}_{\overline{\mathcal{O}}(1)}(\mathrm{OG})$ is a number in $\sum_{k=1}^{2 n} \frac{1}{2 k} \mathbb{Z}$.

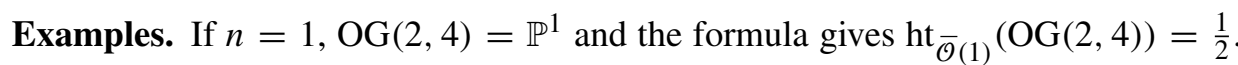
For $n=2$ we have $\mathcal{E}(2)=\left\{[0,0]_{2},[1,0]_{2}\right\}=\{(2,1,1),(2,2)\}$ and the relevant $g$ numbers are $g^{(2,1,1)}=2, g^{(2,2)}=1$, and $g^{(3,1)}=2$. Theorem 4 now gives

$$
\mathrm{ht}_{\overline{\mathcal{O}}(1)}(\mathrm{OG}(3,6))=\frac{1}{2}\left(\mathscr{H}_{1}+\mathscr{H}_{3}\right)+\frac{1}{2} \mathscr{H}_{2}=\frac{13}{6}
$$

which coincides with the Faltings height of $\mathbb{P}^{3}$, as expected. The case of $n=3$ gives the height of the six dimensional quadric $\mathrm{OG}(4,8)$. The required $g$ numbers are

$$
g^{(3,2,1,1)}=8, \quad g^{(3,2,2)}=3, \quad g^{(3,3,1)}=4, \quad g^{(2,2,2,1)}=1, \quad g^{(4,2,1)}=7 .
$$

It follows that

$$
\mathrm{ht}_{\overline{\mathcal{O}}(1)}(\mathrm{OG}(4,8))=2 \mathscr{H}_{1}+3 \mathscr{H}_{3}+2 \mathscr{H}_{5}=\frac{181}{15} .
$$


This agrees with the known result from [CM, Cor. 2.2.10]. Finally, when $n=4$ we find (using the computer program Magma) that

$$
\begin{gathered}
g^{(4,3,2,1,1)}=88, \quad g^{(4,3,2,2)}=30, \quad g^{(4,3,3,1)}=36, \quad g^{(4,4,2,1)}=41, \\
g^{(4,2,2,2,1)}=22, \quad g^{(3,3,3,2)}=8, \quad g^{(5,3,2,1)}=66,
\end{gathered}
$$

and therefore

$$
\mathrm{ht}_{\overline{\mathcal{O}}(1)}(\mathrm{OG}(5,10))=\frac{1}{2}\left(44 \mathscr{H}_{1}+19 \mathscr{H}_{3}+33 \mathscr{H}_{4}+28 \mathscr{H}_{5}+41 \mathscr{H}_{7}\right) .
$$

\section{A comparison of height formulas}

In this section we finish the proof of Theorem 1 by computing the exact value of the constant $r_{n}$ in (7). This will be done by comparing the height formula derived in the last section with the corresponding formula from [T4]. If we substitute $r_{n}$ in place of $\mathscr{H}_{n} / 2$ in the last relation $\mathcal{R}_{2}$ of $\S 2$ and carry out the subsequent analysis in $\S 3$ and $\S 4$, we arrive at the following formula for the height of OG under its fundamental embedding:

$$
\mathrm{ht}_{\overline{\mathcal{O}}(1)}(\mathrm{OG})=\frac{1}{2} \sum_{\substack{0 \leqslant a+2 b<n \\ a, b \geqslant 0}}(-1)^{b} 2^{-\delta_{a 0}} \mathscr{H}_{2 a+2 b+1} g^{[a, b]_{n}}+\frac{r_{n}}{2} g^{(n+1, \rho(n-1))} .
$$

For each $a, b \geqslant 0$ with $a+2 b<n$, let $\langle a, b\rangle_{n}$ denote the partition in $D_{n}$ whose set of parts is $\{1, \ldots, n\} \backslash\{a+2 b+1, a\}$. The more complicated formula for the Faltings height of OG obtained in [T4, Thm. 6] states that

$$
\begin{aligned}
\mathrm{ht}_{\overline{\mathcal{O}}(1)}(\mathrm{OG})=\frac{n d}{4} g^{\rho(n)}+\frac{1}{2} \sum_{\substack{0 \leqslant a+2 b<n \\
a, b \geqslant 0}}(-1)^{b} 2^{-\delta_{a 0}} \mathcal{H}_{2 a+2 b+1} g^{[a, b]_{n}} \\
-\frac{1}{2} \sum_{\substack{0 \leqslant a+2 b<n \\
a, b \geqslant 0}} \frac{(-1)^{a} 2^{-\delta_{a 0}}}{a+b+1}\left(\begin{array}{c}
d \\
2 a+2 b+2
\end{array}\right) g^{\langle a, b\rangle_{n}} .
\end{aligned}
$$

Subtracting (27) from the previous equation, we obtain

$$
\frac{n d}{2} g^{\rho(n)}-r_{n} g^{(n+1, \rho(n-1))}=\sum_{\substack{0 \leqslant a+2 b<n \\
a, b \geqslant 0}} \frac{(-1)^{a} 2^{-\delta_{a 0}}}{a+b+1}\left(\begin{array}{c}
d \\
2 a+2 b+2
\end{array}\right) g^{\langle a, b\rangle_{n}} .
$$

We are fortunate that all of the $g$ numbers in (28) are indexed by strict partitions, and hence may be computed using formula (16). This gives

$$
g^{(n+1, \rho(n-1))}=\frac{d}{2} g^{\rho(n)}
$$


and, setting $(s, t)=(a+2 b+1, a)$,

$$
\left(\begin{array}{c}
d \\
s+t+1
\end{array}\right) g^{\langle a, b\rangle_{n}}=\frac{d}{4(s+t+1)} \frac{s-t}{s+t} \frac{(n+s) !(n+t) !}{(s+t) ! s ! t !(n-s) !(n-t) !} g^{\rho(n)} .
$$

Using (29) and (30) in (28), we deduce that the desired equation $r_{n}=\mathscr{H}_{n} / 2$ is equivalent to the identity displayed in the next Proposition.

\section{Proposition 2.}

$$
\sum_{\substack{0 \leqslant t<s \leqslant n \\ s+t \text { odd }}} \frac{(-1)^{t}}{(s+t+1)^{2}} \frac{s-t}{s+t} \frac{(n+s) !(n+t) !}{(s+t) ! s ! t !(n-s) !(n-t) !}=n-\frac{1}{2} \mathscr{H}_{n} .
$$

Proof. Let $a_{n}$ denote the left hand side of (31). For $n>1$, we have

$$
a_{n}-a_{n-1}=2 n \sum_{\substack{0 \leqslant t<s \leqslant n \\ s+t \text { odd }}} \frac{(-1)^{t}(s-t)(n-1+s) !(n-1+t) !}{(s+t+1)^{2}(s+t) ! s ! t !(n-s) !(n-t) !} .
$$

Hence it will suffice to prove that, for $n>1$,

$$
S:=\sum_{\substack{0 \leqslant t<s \leqslant n \\ s+t \text { odd }}} \frac{(-1)^{t}(s-t)(n-1+s) !(n-1+t) !}{(s+t+1)^{2}(s+t) ! s ! t !(n-s) !(n-t) !}=\frac{1}{2 n}\left(1-\frac{1}{2 n}\right) .
$$

We now substitute $s=u-t$ in the above summand and express the sum over $t$ using hypergeometric notation. Observe that this introduces an extraneous term of $1 / n^{2}$ when $u=-1$. We obtain the equation

$$
S+\frac{1}{n^{2}}=\sum_{u \text { odd }} \frac{u \Gamma(n+u)}{n \Gamma(u+2)^{2} \Gamma(n+1-u)} 4 F_{3}\left(\begin{array}{c}
-u, 1-u / 2,-n, n \\
-u / 2,1-u+n, 1-u-n
\end{array} \mid 1\right) .
$$

The identity from $[\mathrm{B}, \S 4.3,(3)]$ for a well-poised ${ }_{5} F_{4}$ implies that

$$
{ }_{4} F_{3}\left(\begin{array}{c|c}
-u, 1-u / 2,-n, n \\
-u / 2,1-u+n, 1-u-n
\end{array} \mid 1\right)=\frac{(1-u)_{n}\left(\frac{1}{2}+\frac{u}{2}\right)_{n}}{(u)_{n}\left(\frac{1}{2}-\frac{u}{2}\right)_{n}},
$$

where $(x)_{n}=x(x+1) \cdots(x+n-1)$ is the Pochhammer symbol. Now substitute (32) into the previous formula for $S$, set $u=2 v-1$, and express the result as a hypergeometric series. We obtain that

$$
4 n^{2} S+1={ }_{3} F_{2}\left(\begin{array}{c|c}
n,-n,-\frac{1}{2} & 1 \\
1, \frac{1}{2} & 1
\end{array}\right) .
$$


The ${ }_{3} F_{2}$ transformation formula

${ }_{3} F_{2}\left(\begin{array}{c|c}-n, a, b & 1 \\ c, d & 1\end{array}\right)=\frac{(c-a)_{n}(d-a)_{n}}{(c)_{n}(d)_{n}} \cdot{ }_{3} F_{2}\left(\begin{array}{c}-n, a, a+b-c-d+1-n \\ a-d+1-n, a-c+1-n\end{array} \mid 1\right)$

with $a=-1 / 2, b=n, c=1$, and $d=1 / 2$ gives

$$
\begin{gathered}
{ }_{3} F_{2}\left(\begin{array}{c}
n,-n,-\frac{1}{2} \\
1, \frac{1}{2}
\end{array} \mid 1\right)=\frac{\left(\frac{3}{2}\right)_{n}}{\left(\frac{1}{2}\right)_{n}} \cdot 3 F_{2}\left(\begin{array}{c}
-n,-1,-\frac{1}{2} \\
-n,-\frac{1}{2}-n
\end{array} \mid 1\right) \\
=(2 n+1) \cdot{ }_{2} F_{1}\left(\begin{array}{c}
-1,-\frac{1}{2} \\
-\frac{1}{2}-n
\end{array}\right)=2 n,
\end{gathered}
$$

where the last equality follows by Gauss's hypergeometric theorem. Therefore

$$
S=\frac{1}{2 n}-\frac{1}{4 n^{2}},
$$

as desired.

Guoce Xin has pointed out a different proof of (31), using a variant of Zeilberger's creative telescoping method [PWZ, §6]. Let

$$
F(n, s, t)=\frac{(-1)^{t}}{(s+t+1)^{2}} \frac{s-t}{s+t} \frac{(n+s) !(n+t) !}{(s+t) ! s ! t !(n-s) !(n-t) !} .
$$

Applying Zeilberger's algorithm, one shows that the second difference

$$
\Delta^{2} F(n, s, t)=F(n+2, s, t)-2 F(n+1, s, t)+F(n, s, t)
$$

satisfies

$$
\Delta^{2} F(n, s, t)=G(n, s, t)-G(n, s+1, t)+H(n, s, t)-H(n, s, t+1),
$$

where

$$
G(n, s, t)=(-1)^{t} \frac{A}{N} \frac{s}{s+t} \frac{(n+s) !(n+t) !}{(s+t) ! s ! t !(n+2-s) !(n+2-t) !}
$$

and

$$
H(n, s, t)=(-1)^{t} \frac{B}{N} \frac{t}{s+t} \frac{(n+s) !(n+t) !}{(s+t) ! s ! t !(n+2-s) !(n+2-t) !} .
$$

Here $N=(n+1)(n+2)$ and

$$
\begin{aligned}
& A=N^{2}+2 t(t-s) N+t(1-t)\left(s^{2}+t\right) \\
& B=N^{2}+\left(2 s^{2}-2 t^{2}-4 s t+s+t\right) N+(t-1)\left(2 s^{3}+s^{2} t-s^{2}+t^{2}+s t\right) .
\end{aligned}
$$


Equation (33) is checked by dividing both sides by $F(n, s, t)$, and verifying the resulting identity of rational functions. Let $b_{n}=2 n-\mathscr{H}_{n}$. To prove (31), it suffices to show that

$$
2 \Delta^{2} a_{n}=\Delta^{2} b_{n}=\frac{1}{N} .
$$

Equation (34) is proved by summing (33) over all $s, t$ with $0 \leqslant s, t \leqslant n+2$ and noting that

$$
\left.(G(n, s, t)+H(n, s, t))\right|_{s=t=0}=\frac{1}{N} .
$$

Remark. It would be interesting to have a direct computation of the constant $r_{n}$, for instance by evaluating the Bott-Chern form of the exact sequence (1), or an analogous sequence over isotropic flag bundle of $E \rightarrow$ OG. Although more elementary than the above comparison of heights, the combinatorial analysis required by such a direct approach appears difficult.

\section{References}

[B] W. N. Bailey, Generalized Hypergeometric Series. Cambridge Tracts Math. Math. Phys. 32, Cambridge University Press, Cambridge 1935. Zbl 0011.02303 MR 0185155

[Bo] A. Borel, Sur la cohomologie des espaces fibrés principaux et des espaces homogènes de groupes de Lie compacts. Ann. of Math. 57 (1953), 115-207. Zbl 0052.40001 MR 0051508

[BGS] J.-B. Bost, H. Gillet and C. Soulé, Heights of projective varieties and positive Green forms. J. Amer. Math. Soc. 7 (1994), 903-1027. Zbl 0973.14013 MR 1260106

[BC] R. Bott and S. S. Chern, Hermitian vector bundles and the equidistribution of the zeroes of their holomorphic sections. Acta. Math. 114 (1968), 71-112. Zbl 0148.31906 MR 0185607

[C] É. Cartan, Sur une classe remarquable d'espaces de Riemann I; II. Bull. Soc. Math. France 54 (1926), 214-264; 55 (1927), 114-134; also in Oeuvres Complètes, Partie I, Vol. 2, Gauthier-Villars, Paris 1952, 587-659. JFM 53.0390.01 MR 1504900 MR 1504909

[CM] J. Cassaigne and V. Maillot, Hauteur des hypersurfaces et fonctions zêta d'Igusa. $J$. Number Theory 83 (2000), 226-255. Zbl 1001.11027 MR 1772614

[F] G. Faltings, Diophantine approximation on abelian varieties. Ann. of Math. 133 (1991), 549-576. Zbl 0734.14007 MR 1109353

[GS1] H. Gillet and C. Soulé, Arithmetic intersection theory. Inst. Hautes Études Sci. Publ. Math. 72 (1990), 93-174. Zbl 0741.14012 MR 1087394

[GS2] H. Gillet and C. Soulé, Characteristic classes for algebraic vector bundles with hermitian metrics, I; II. Ann. of Math. 131 (1990), 163-203; 205-238. Zbl 0715.14018 MR 1038362 Zbl 0715.14006 MR 1043268 
[HB] H. Hiller and B. Boe, Pieri formula for $\mathrm{SO}_{2 n+1} / \mathrm{U}_{n}$ and $\mathrm{Sp}_{n} / \mathrm{U}_{n}$. Adv. Math. 62 (1986), 49-67. Zbl 0611.14036 MR 0859253

[J] J. C. Jantzen, Representations of Algebraic Groups. Second edition, Math. Surveys Monogr. 107, Amer. Math. Soc., Providence, RI, 2003. Zbl 1034.20041 MR 2015057

[KK] C. Kaiser and K. Köhler, A fixed point formula of Lefschetz type in Arakelov geometry III: representations of Chevalley schemes and heights of flag varieties. Invent. Math. 147 (2002), 633-669. Zbl 1023.14008 MR 1893008

[KT] A. Kresch and H. Tamvakis, Quantum cohomology of the Lagrangian Grassmannian. J. Algebraic Geom. 12 (2003), 777-810. Zbl 1051.53070 MR 1993764

[M] I. G. Macdonald, Symmetric Functions and Hall Polynomials. Second edition, Oxford Math. Monogr., Clarendon Press, Oxford 1995. Zbl 0899.05068 MR 1354144

[Ma] V. Maillot, Un calcul de Schubert arithmétique. Duke Math. J. 80 (1) (1995), 195-221. Zbl 0867.14024 MR 1360616

[PWZ] M. Petkovšek, H. S. Wilf, and D. Zeilberger, $A=B$. A. K. Peters, Ltd., Wellesley, MA, 1997. Zbl 0848.05002 MR 1379802

[P] P. Pragacz, Algebro-geometric applications of Schur $S$ - and $Q$-polynomials. In Topics in invariant theory (Paris, 1989/1990), Lecture Notes in Math. 1478, Springer-Verlag, Berlin 1991, 130-191. Zbl 0783.14031 MR 1180989

[PR] P. Pragacz and J. Ratajski, Formulas for Lagrangian and orthogonal degeneracy loci; $\widetilde{Q}$-polynomial approach. Compositio Math. 107 (1) (1997), 11-87. Zbl 0916.14026 MR 1457343

[S] I. Schur, Über die Darstellung der symmetrischen und der alternierenden Gruppe durch gebrochene lineare Substitutionen. J. Reine Angew. Math. 139 (1911), 155-250. JFM 42.0154.02

[T1] H. Tamvakis, Bott-Chern forms and arithmetic intersections. Enseign. Math. 43 (1997), 33-54. Zbl 0917.32025 MR 1460121

[T2] H. Tamvakis, Schubert calculus on the arithmetic Grassmannian. Duke Math. J. 98 (1999), 421-443. Zbl 0989.14007 MR 1695796

[T3] H. Tamvakis, Arakelov theory of the Lagrangian Grassmannian. J. Reine Angew. Math. 516 (1999), 207-223. Zbl 0934.14018 MR 1724621

[T4] H. Tamvakis, Height formulas for homogeneous varieties. Michigan Math. J. 48 (2000), 593-610. Zbl 1077.14527 MR 1786507

Received January 2, 2004

Harry Tamvakis, University of Maryland, Department of Mathematics, 1301 Mathematics Building, College Park, MD 20742-4015, U.S.A.

E-mail: harryt@math.umd.edu 